\title{
EVITA Project: Comparison Between Traditional Non-Destructive Techniques and Phase Contrast X-Ray Imaging Applied to Aerospace Carbon Fibre Reinforced Polymer
}

\author{
Matthieu Gresil ${ }^{1}$ (D) - Vincent Revol ${ }^{2}$. \\ Konstantinos Kitsianos ${ }^{3}$ - Georges Kanderakis ${ }^{4}$. \\ Ilias Koulalis ${ }^{4}$ - Marc-Olivier Sauer $^{3}$ - Hervé Trétout ${ }^{5}$. \\ Ana-Maria Madrigal ${ }^{2}$
}

Received: 8 September 2016 / Accepted: 3 October 2016 /Published online: 15 October 2016

(C) The Author(s) 2016. This article is published with open access at Springerlink.com

\begin{abstract}
The EU-project EVITA (Non-Destructive EValuation, Inspection and Testing of Primary Aeronautical Composite Structures Using Phase Contrast X-Ray Imaging) aims at bringing Grating-based Phase Contrast X-ray imaging technology to Non-Destructive Evaluation and Inspection of advanced primary and/or complex aerospace composite structures. Grating-based Phase Contrast X-Ray Imaging is based on the so-called Talbot-Lau interferometer, which is made of the combination of a standard X-ray apparatus with three transmission gratings as documented in the literature. This paper presents a comparison of two traditional non-destructive techniques (NDT): ultrasonic through transmission (immersed and water jet) and ultrasonic phased-array pulse echo, with the developed phase contrast XRay Imaging applied to advanced aerospace carbon fibre reinforced polymer. Typical defects produced during manufacture is examined as part of the testing and validation procedure. The following defects have been identified as being those most likely to be detected more effectively by the Grating-based Phase Contrast X-Ray Imaging process than other state of the art industrial NDT techniques: porosity, foreign objects, cracks, resin rich, cut fibres, and wavy fibres. The introduction of this innovative methodology is expected to provide the aeronautical industry with a reliable and detailed insight of the integrity of thin and thick
\end{abstract}

Matthieu Gresil

matthieu.gresil@manchester.ac.uk

1 i-Composites Lab, School of Materials, University of Manchester, Manchester, UK

2 Centre Suisse d'Électronique et Microtechnique - CSEM, Neuchatel, Switzerland

3 GMI Aero, Paris, France

4 National Technical University of Athens, Athens, Greece

5 DASSAULT Aviation, Argenteuil, France 
composite structures as well as of complex geometry ones, such as integrated closed boxes and sandwiches.

Keywords X-Ray imaging · Phase contrast · Porosity · Cracks · Image fusion

\section{Introduction}

Grating-based Phase Contrast X-Ray Imaging is based on the so-called Talbot-Lau interferometer, which is made of the combination of a standard X-ray apparatus with three transmission gratings as documented in the literature [1-4]. The method derives its potential from the fact that three different contrast mechanisms are combined in a single measurement. Indeed, not only the conventional absorption image can be extracted but also the refraction image (also called differential phase contrast image) and the scattering image (also called dark field image), which are related respectively to the refraction of the $\mathrm{X}$-ray beam inside the sample and to the ultra-small angle scattering caused by its microstructure. Preliminary studies have shown that the scattering image provides a powerful tool to detect any change in the arrangement of the fibres due to the presence of defects such as porosity, fiber waviness, micro-cracks or resin rich/resin poor areas [5-10].

Within the project EVITA (www.evita-project.eu), the requirements and needs of the aeronautics industry in terms of the non-destructive inspection of thick and thin composite components were collected and analysed. From there, a customized demonstrator was designed and realized in order to benchmark this novel technology against the following non-destructive inspection (NDI) techniques: water jet ultrasonic, phased array ultrasonic, thermography and computed tomography.

\section{Imaging Principle}

At low energies, the interaction of X-rays with matter can be described by a complex refractive index $[11,12]$ :

$$
n=1-\delta-i \beta
$$

Where $(1-\delta)$ and $\beta$ are the real and the imaginary part of the complex refraction index, respectively. The imaginary part $\beta$ describes the attenuation of the X-rays in matter and is related to the linear absorption coefficient $\mu_{1}(E)$ at energy $E$ by:

$$
\mu_{1}(E)=\frac{4 \pi \beta(E)}{\lambda}
$$

Where $\lambda$ is the wavelength of the X-rays. In conventional X-ray testing, the attenuation of an X-ray beam by the sample under test is measured and is given by $T(E)=\exp \left(-\mu_{1}(E) \times t\right)$ for an homogeneous sample of thickness $t$. Recently, a technique called phase contrast X-ray imaging was invented, which allows for the measurement of changes of the phase front of the X-ray waves as shown in Fig. 1. 


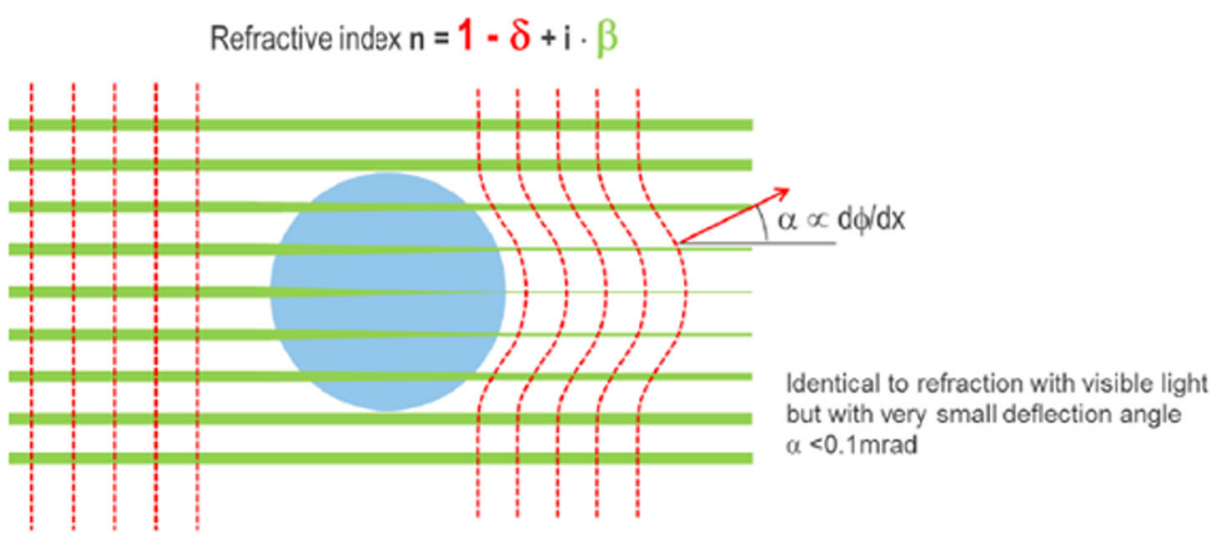

Fig. 1 In conventional X-ray imaging, the attenuation of the X-ray beam due to its absorption in the sample is measured and is related to the attenuation coefficient $\beta$. On the contrary, in phase contrast $\mathrm{X}$-ray imaging, changes in the phase front of the beam are measured which are linked to the refraction coefficient $\delta$

The phase shift of the X-ray wave inside the sample is given by:

$$
\phi(E)=\frac{2 \pi}{\lambda} \delta(E) \times t
$$

Since the refraction coefficient can be up to three orders of magnitude large than the attenuation coefficient, a better contrast can be achieved between similar materials (e.g. two different polymers). This is especially true for light materials, such as biological soft tissues or plastics, and this relative increases with the X-ray photon energy.

The phase contrast X-ray imaging system developed here is based on the Talbot-Lau grating interferometer [4]. The grating interferometer consists of a conventional X-ray source, a commercial X-ray detector and three X-ray gratings G0, G1, and G2 as shown in Fig. 2a. The phase sensitive part consists of the beam-splitter grating G1 and the analyser grating G2. G1 is a phase grating, which induces periodical phase modulation on the X-ray wave front and acts as a beam-splitter. The divided beams then interfere downstream of G1 and result in an intensity pattern $I_{f r}$ in the plane G2. G2 is an absorbing mask with a periodicity matching that of the interference fringes. Depending on the position of G2 relative to the interference pattern, a varying amount of radiation is transmitted and recorder by the X-ray detector. Thus, for a given position of $\mathrm{G} 2$, any change in the intensity modulations (for example, of the mean, amplitude, or position) locally induces a change of the intensity $I_{p i x}$ recorded by the pixel (r,c) of the X-ray detector, where $\mathrm{r}$ and $\mathrm{c}$ are integers indicating the pixel position (row and column). For polychromatic radiation, the intensity $I_{p i x}$ can be expressed as

$$
I_{p i x}(r, c)=Q_{0}(r, c) \times\left[1+\nu(r, c) \cdot \sin \left(\psi_{1}(r, c)\right)\right]
$$

Where $Q_{0}$ is the mean intensity (averaged over $\psi_{1} \in[0,2 \pi]$ ) received by the detector pixel, which depends on the X-ray flux and the absorption of the gratings. $\nu$ is the visibility of the interference, which ranges from 0 to 1 indicates the quality of the interference fringes. $\nu=0$ means that there is no interference at all, while $\nu=1$ is the ideal case (only achievable for perfect gratings and monochromatic radiation) The phase coefficient $\psi_{1}$ is related to the relative position of $\mathrm{G} 2$ with respect to the interference. For example, it is equal to 0 when 


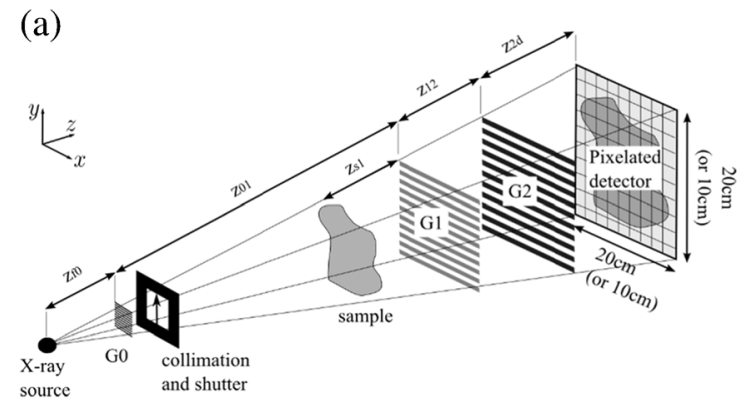

(b)

Fig. 2 a Schematic of the grating-based Phase Contrast X-Ray Imaging system and $\mathbf{b}$ cross sectional view of the interactions of the X-ray beam with the sample

the interference maxima are aligned with the absorbing part of G2. Inversely, $\psi_{1}$ amounts to $\pi / 2$ when the maxima are aligned with the transmitting part of $\mathrm{G} 2$.

In the presence of a sample, the measured pixel intensity will be changed due to three different effects, as shown in Fig. 2b. For convenience, the subscript " $r$ " and " $s$ " to refer to the measurement done without sample ("reference") and with sample are used, respectively.

First, the attenuation of the X-ray beam due to photoelectric absorption and Compton scattering at large angles leads to a decrease of the mean intensity $Q_{0}$ of the interference fringes. Secondly, the beam is refracted by an angle $\alpha$ and this results in a local displacement (along the y-direction) of the fringes and thus a change of the phase coefficient $\psi_{1}$. Finally, in the presence of inhomogeneity (porosity, cracks, etc.) at the microscopic level $(0.1$ to $50 \mu \mathrm{m})$, the beam is scattered at small angles and a loss of visibility $\nu$ of the interference fringes ensues. The different contributions can be decoupled using different imaging modes and thus three images can be reconstructed, namely the absorption image $T$, the differential phase contrast image $D P$, and the scattering image $V$ :

$$
T(r, c)=\frac{Q_{0}^{s}(r, c)}{Q_{0}^{r}(r, c)}, D P(r, c)=\frac{\psi_{1}^{s}(r, c)-\psi_{1}^{r}(r, c)}{2 \pi}, V(r, c)=\frac{\nu^{s}(r, c)}{\nu^{r}(r, c)}
$$

For example, Fig. 3 shows the results of the reconstruction of the three images $T, D P$, and $V$ for a carbon fibre composite sample.

It is important to note that both the refraction angle and the small angle scattering are measured only along the transverse direction, perpendicular to the gratings' lines. This means that anisotropic defects, such as cracks running along the other direction, parallel to the gratings' lines may not be seen in the images. In order to solve this problem and see defects
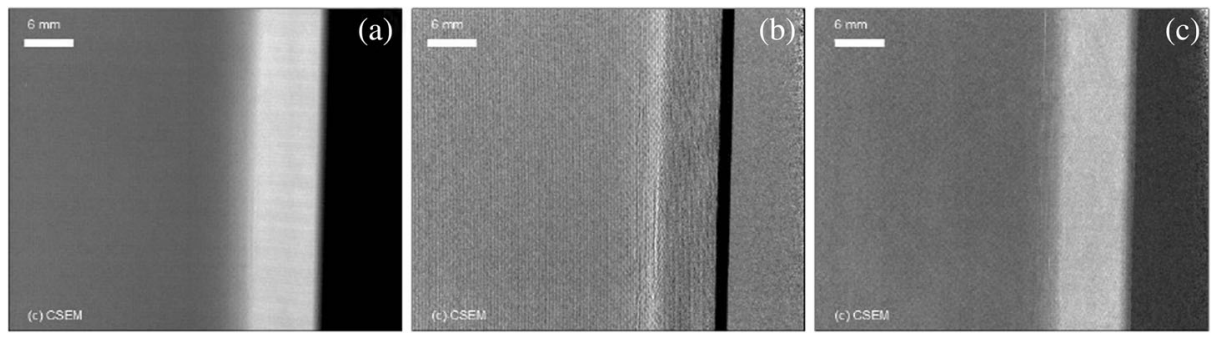

Fig. 3 Example of a absorption; $\mathbf{b}$ differential phase contrast and $\mathbf{c}$ scattering images obtained for a curved fibre reinforced polymer 
in all directions, the gratings can be rotated by $90^{\circ}$ and another image can be acquired, which will be sensitive in the perpendicular direction.

Actually, the anisotropic sensitivity of the grating interferometer can even be used to extract directional information on the sample, such as the number of plies orientated along a given direction. Flaws such as wavy fibres are then easily detectable using this directional sensitivity [9].

In order to use this advantage, the grating interferometer must be designed to allow the rotation of the ensemble $(\mathrm{G} 1+\mathrm{G} 2)$ independently of the detector, in such a way that images of the sample can be acquired for different directions of the sensitivity. More details can be found in the literature $[6,7]$.

\section{Experiments}

\subsection{Grating-Based Phase Contrast X-Ray Imaging (XPCI)}

A schematic view of the grating-based Phase Contrast X-Ray Imaging system is shown in Fig. 2a. It consists of a standard high power X-ray source (Varian HPW-160-11) and pixelated detector array (Dexela DEX2315) coupled to three gratings forming an X-ray interferometer. The principle of the X-ray grating interferometer is explained in details in the literature [1-3, 13]. A collimator made out of lead is used to control the illuminated area while a shutter allows to block the X-ray beam during the idle time of the detector. In the present configuration, the sample can be moved independently using an XY gantry (IAI axis).

The gratings were manufactured at CSEM using MEMS fabrication processes on Silicon wafers of diameter $150 \mathrm{~mm}$, which allows to achieve a grating size of $100 \times 100 \mathrm{~mm}$ on a single wafer. The parameters of the demonstrator are summarized in Table 1.

Three images are obtained using the EVITA demonstrator, namely the absorption, refraction and scattering images. The absorption image corresponds to the conventional X-ray image, except that the blurring effect due to the Compton scattering is suppressed in the direction perpendicular to the grating lines. The absorption image is related to the attenuation coefficient of the material and its thickness.

The refraction image is proportional to the refraction angle measured pixel-wise by the interferometer. The refraction angle is linked to the derivative of the phase shift in the direction perpendicular to the grating lines (direction $y$ in Fig. 2a). In contrast to the absorption image, the refraction image is thus related to the refraction coefficient as well as to the thickness of the sample [4].

Table 1 Key parameters of the EVITA demonstrator

\begin{tabular}{ll}
\hline & Parameters \\
\hline Source acceleration voltage & $40-70 \mathrm{kV}$ \\
Maximal sample thickness (CFRP) & $50 \mathrm{~mm}$ \\
System length (source to detector) & $1.45 \mathrm{~m}$ \\
Measurement area (stitching mode) & $1 \times 0.5 \mathrm{~m}^{2}$ \\
Image size (single field) & $7 \times 7 \mathrm{~cm}^{2}$ \\
Effective pixel size & $50-60 \mu \mathrm{m}$ \\
\hline
\end{tabular}


Finally, the scattering image is related to the ultra-small angle X-ray scattering of the beam inside the sample. It has been shown that the USAXS can be expressed in terms of variations of the electronic density of the material at the microscopic level [14]. The scattering image is thus a perfect tool to probe the microscopic texture of composite materials and detect porosity, cracks and variations of the fibre density or orientation [15].

The images were reconstructed using the phase stepping method [13], where the phase stepping was achieved by translation of the grating G2. The X-ray tube source was set to the small focal spot $\left(0.4 \times 0.4 \mathrm{~mm}^{2}\right)$ with the acceleration voltage at $60 \mathrm{kVp}$ and the anode current at $10 \mathrm{~mA}$. 19 phase steps were acquired over 4 periods with an individual exposure time of $750 \mathrm{~ms}$. The measurement was repeated 4 times and averaged. The measurement time for a single field inclusive reconstruction amounts to about $60 \mathrm{~s}$.

\subsection{Materials}

The samples were made out of Epoxy-carbon prepreg (HexPly 914C-T300H(6 K)-5-34 \%). Two thickness composite materials were made, 4 and $20 \mathrm{~mm}$. The results presented here were obtained with a 32 and 160 plies quasi-isotropic lay-up $\left(+45^{\circ} / 90 \%-45^{\circ} / 0^{\circ}\right) 4 \mathrm{~s}$ and $\left(+45^{\circ} / 90^{\circ} \%\right.$ $\left.45^{\circ} / 0^{\circ}\right) 40 \mathrm{~s}$, respectively. In order to test the capability of the PCI technique, manufacturing routes were developed which produced controlled defect in a repeatable manner. Table 2 shows the range of defect reference samples investigated in this publication.

The results obtained with the EVITA demonstrator were benchmarked against four state-ofthe-art NDI methods: Ultrasonic through transmission (UTT) (i.e. water jet and immersed), phased array ultrasonic (PA), and conventional X-Ray computed tomography (X-Ray CT).

In our preliminary work [9], the EVITA demonstrator was benchmarked on two different types of artificial flaws: fiber cut and out-of-plane wrinkle. These two flaws were detected by the EVITA demonstrator with a comparably fast exposure time. No other NDI method used for the benchmarking was able to detect both flaws.

\section{Results and Discussions}

\subsection{Porosity Detection}

Figures 4, 5, and 6 show the results of the comparison for the first samples, where different porosity contents were introduced during the manufacturing process. The porosity can be detected in the scattering image using the EVITA demonstrator as well as with the UTT and PA systems. All other benchmarking methods (i.e. IR-thermography and X-Ray CT) were not able

Table 2 Composite sample list

\begin{tabular}{llll}
\hline Sample ID & Defects & Materials & Comments \\
\hline $137186-14$ & porosity $<2 \%$ & Monolithic, Epoxy-carbon prepreg layup & thick (20 mm) \\
$137186-16$ & porosity 2 to $5 \%$ & Monolithic, Epoxy-carbon prepreg layup & thick $(20 \mathrm{~mm})$ \\
$137186-18$ & porosity $>5 \%$ & Monolithic, Epoxy-carbon prepreg layup & thick $(20 \mathrm{~mm})$ \\
$137186-2$ & cracks & Monolithic, Epoxy-carbon prepreg layup & thin $(4 \mathrm{~mm})$ \\
\hline
\end{tabular}




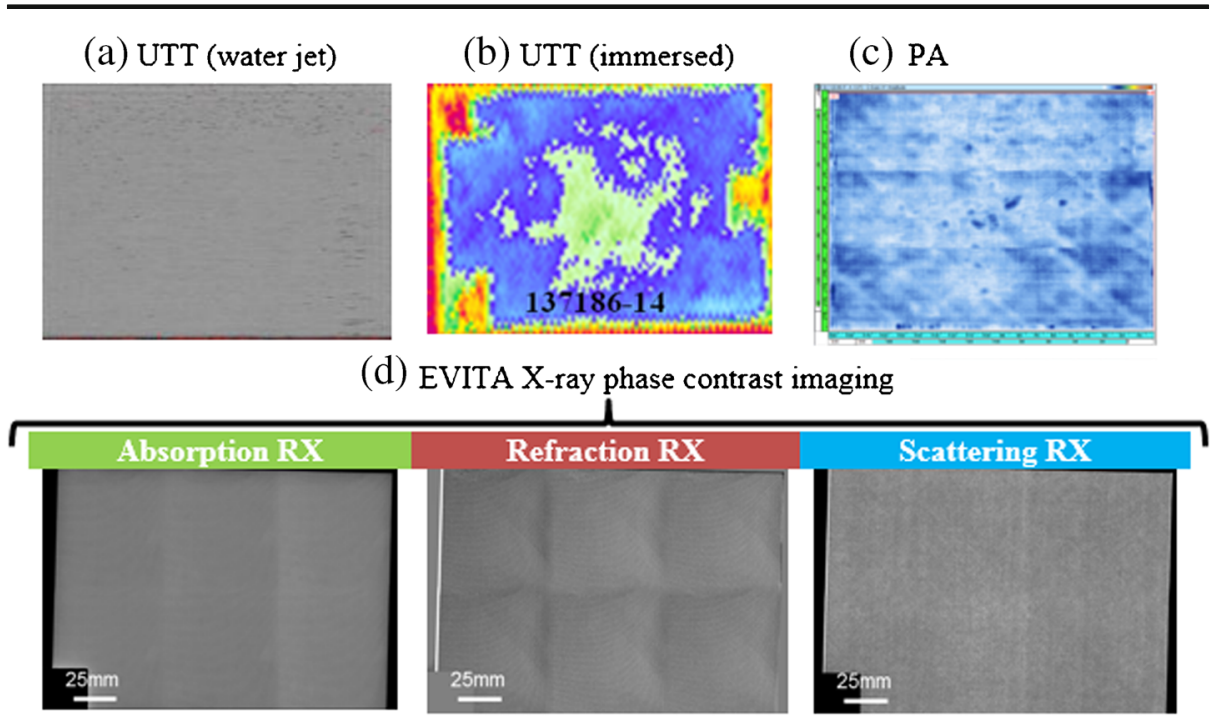

Fig. 4 Sample with porosity $<2 \%$. The images obtained by UTT and PA are shown in (a), (b) and (c). The absorption, refraction and scattering images obtained with the EVITA demonstrator are shown in (d)

to detect the defects. The same colour bar on the scattering image is used for all the samples with porosity. Moreover, Moiré artefacts are observed in the absorption and refraction image.

It was observed that the scattering signal is increasing with the porosity level. Moreover, in the zoom-in of the refraction image, elongated voids can be observed. In order to evaluate quantitatively the relationship, the average and standard deviation of the absorption and scattering images were calculated over a region of $100 \times 100$ pixels (about $6 \times 6 \mathrm{~mm}$ ). The averages of the absorption and scattering images are plotted in Fig 7 as a function of the porosity level, where the standard deviation is represented as the error bar. The signal intensity

(a) UTT (water jet)

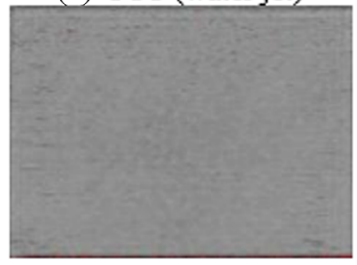

(b) UTT (immersed)

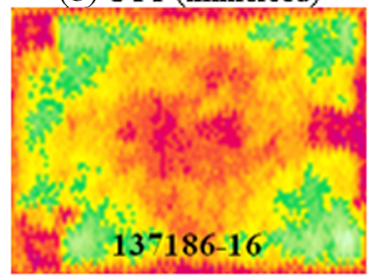

(c) Phased array

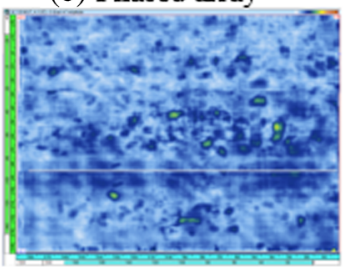

(d) EVITA X-ray phase contrast imaging i

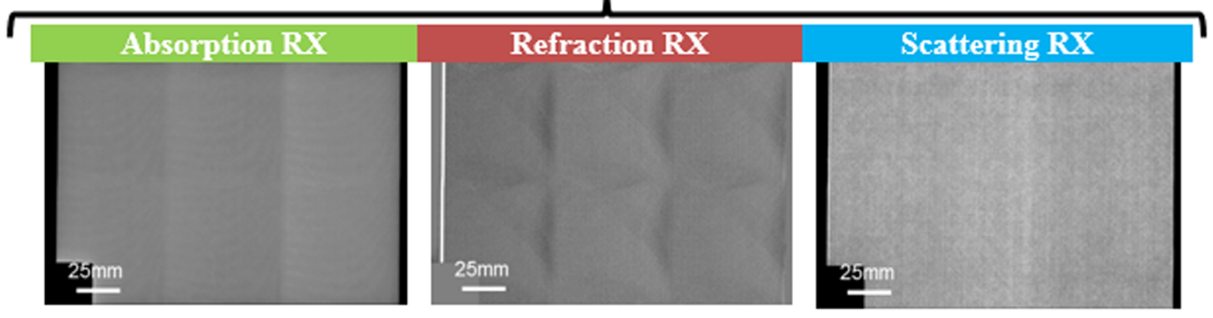

Fig. 5 Sample with porosity 2 to $5 \%$. The images obtained by UTT and PA are shown in (a), (b) and (c). The absorption, refraction and scattering images obtained with the EVITA demonstrator are shown in (d) 
(a) UTT (water jet)

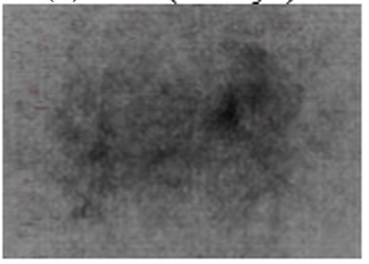

(b) UTT (immersed)

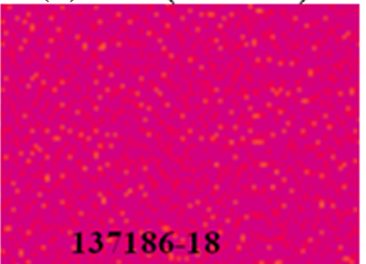

(c) PA

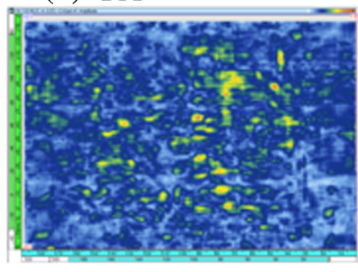

(d) EVITA X-ray phase contrast imaging
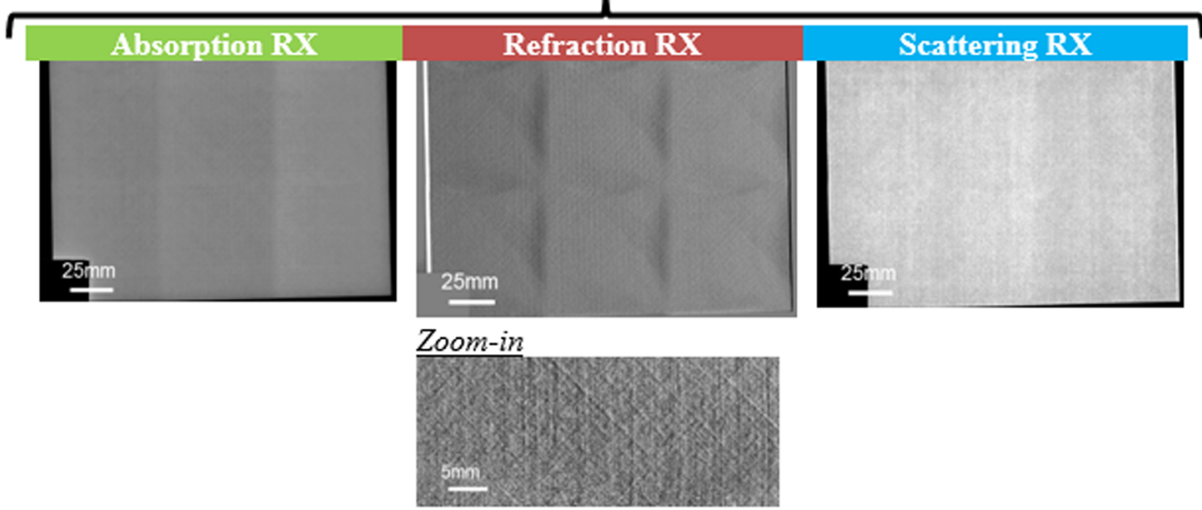

Fig. 6 Sample with porosity $>5 \%$. The images obtained by UTT and PA are shown in (a), (b) and (c). The absorption, refraction and scattering images obtained with the EVITA demonstrator are shown in (d)

of the scattering increase linearly with the level of porosity which is not the case with the absorption. With higher magnification, the EVITA system is able to identify the porosity distribution and size. To obtain the information through the thickness, this system has to be coupled with the computed tomography technique.

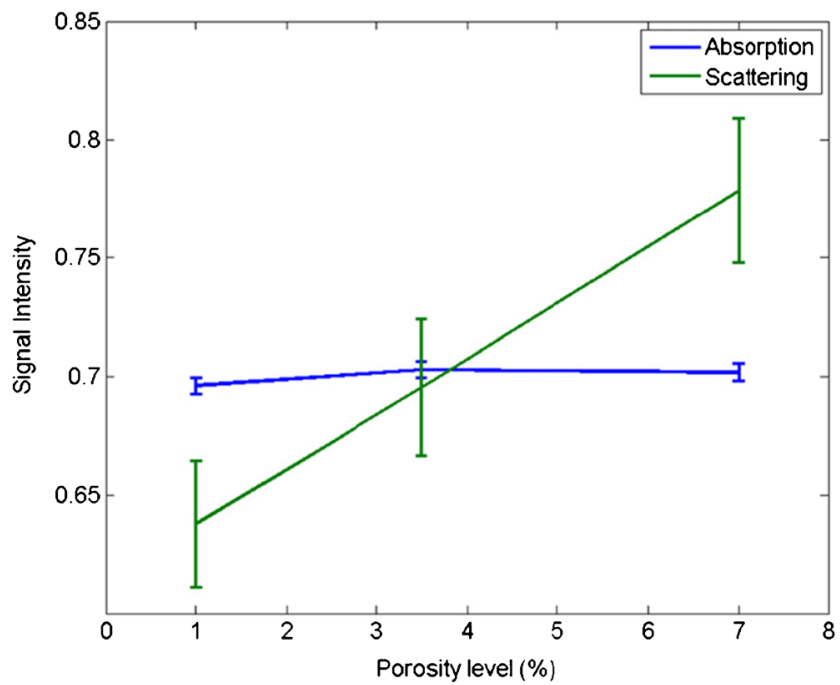

Fig 7 Plots of the average absorption and scattering signal as a function of the porosity for the $20 \mathrm{~mm}$ thick samples. The error bar represents the standard deviation over the region of interest 

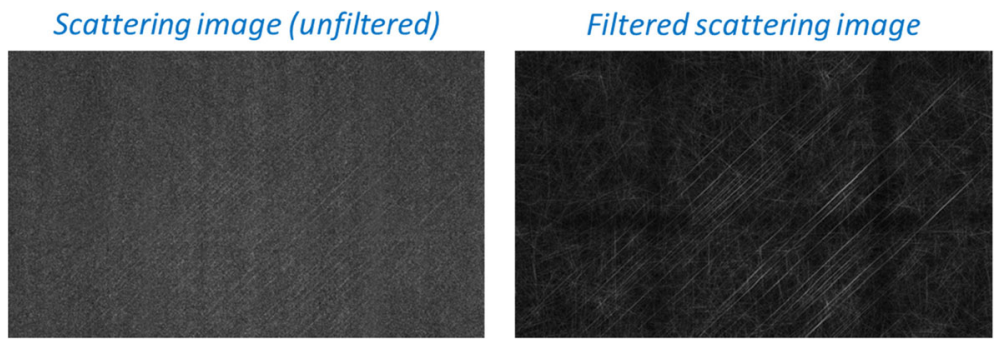

Fig. 8 (left) Scattering image of a sample with micro-cracks and (right) corresponding image after application of the geometrical filter

\subsection{Cracks Detection}

Micro-cracks are usually following the orientation of the fibre layup. For this reason, they display usually a clear orientation (typically $0^{\circ} / 90^{\circ} /+45^{\circ} /-45^{\circ}$ ). A special geometrical filter was developed to enhance the crack signal. The following procedure is implemented: (i) remove low frequency from the image; (ii) filter the image suing ellipsoidal Gauss kernels with angle scanning between 0 and $180^{\circ}$ ( $1^{\circ}$ step); (iii) combine the filtered images by normalising according to the filter response at each angle. An edge along the filter direction will have a greater response than all other edges in the image. And the edges which are not in the direction of the filter will be smoothed out providing a low response close to the average. Figure 8 shows the results of the geometrical filter applied to the scattering images of samples with micro-cracks. This is clear that this geometrical filter strongly enhances the detectability and visibility of the elongated micro-cracks. These measurements demonstrate the ability of the EVITA system to detect micro-cracks in composite components. Moreover, the crack shape and density can be obtained.

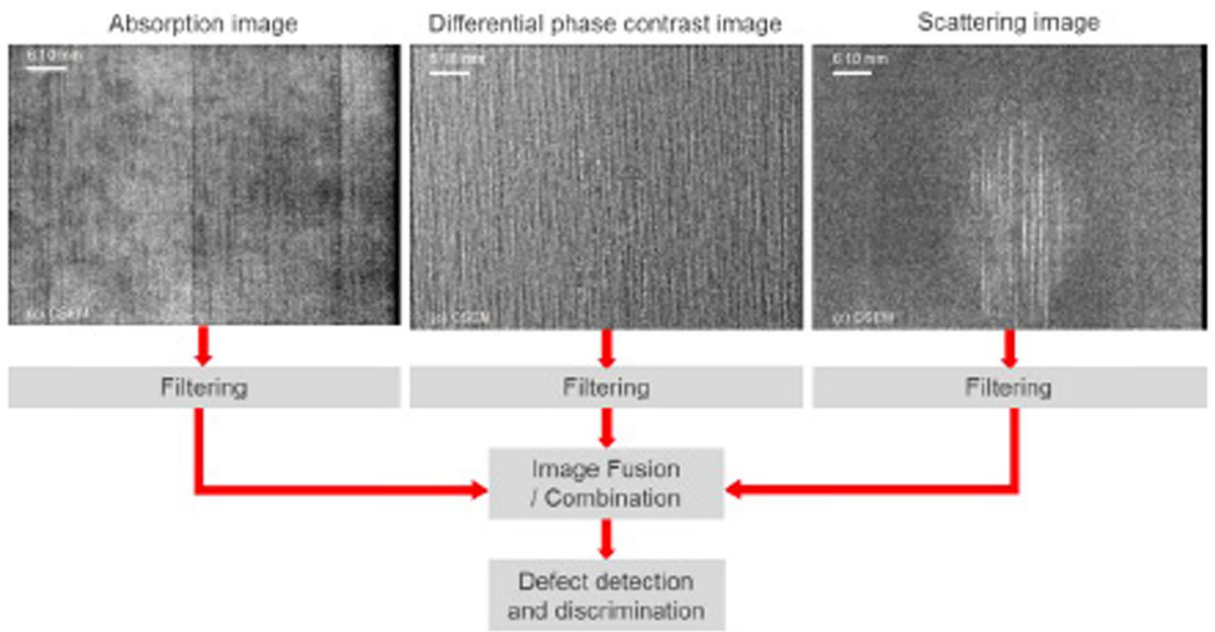

Fig. 9 All three images deliver complementary information, which is combined for defect detection and discrimination 


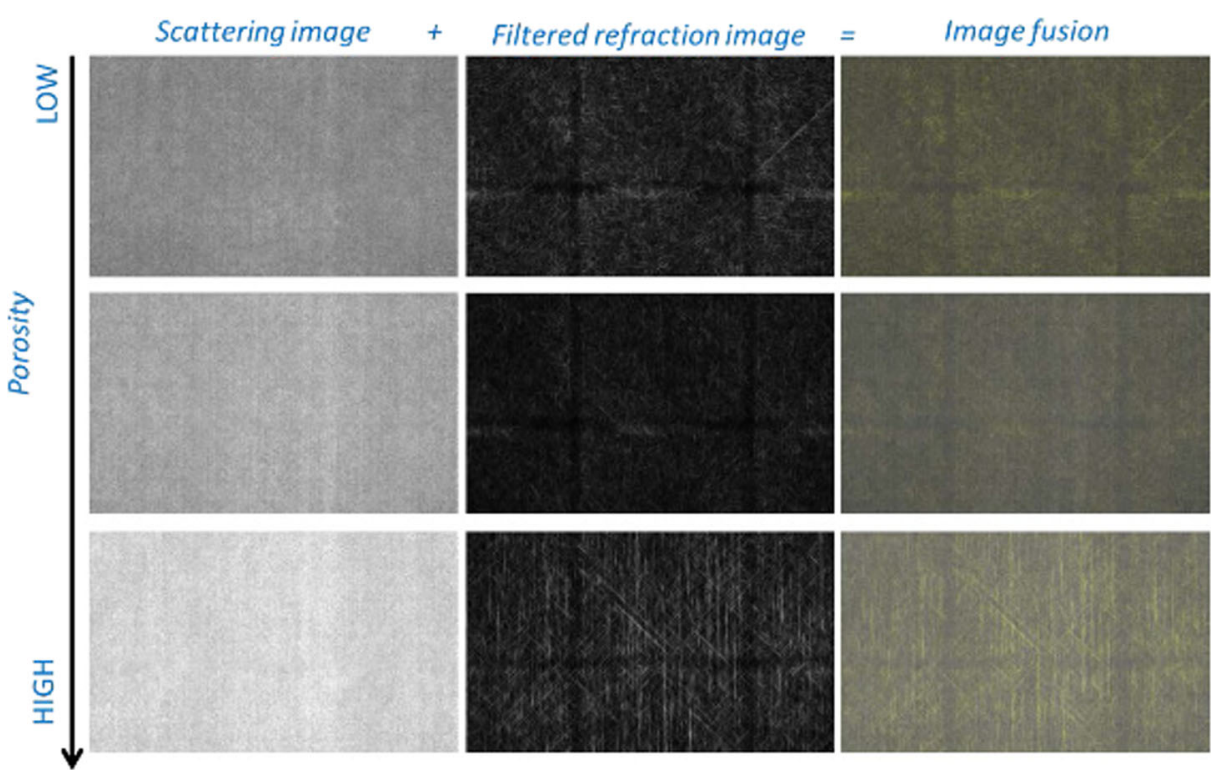

Fig. 10 Example of image fusion of the scattering image with the filtered refraction image for composite samples with different level of porosity $1,3.5$ and $7 \%$

\subsection{Image fusion}

The EVITA phase contrast imaging system aims at detecting and discriminating different defects. As each of the imaging modes (absorption, differential phase contrast (refraction) and small angle scattering) has different sensitivity to different types of defects, the image processing module (IPM) allows to process and combine the different images (Fig. 9). It is thus envisaged that a drop down menu system indicating the defect types under examination in the image processing software will allow the correct combination of images to be obtained which give the best chance of detecting the particular defect type.

The image fusion algorithm aims at visualising both the filtered structure and the background scattering image (unfiltered) at the same time. In the present case, we decided to display the scattering image as grey levels and to overlap the filtered refraction image with yellow colour. Figure 10 displays an example of the image fusion for composite samples with different levels of porosity while Fig. 11 shows the example for a composite sample with micro-cracks.

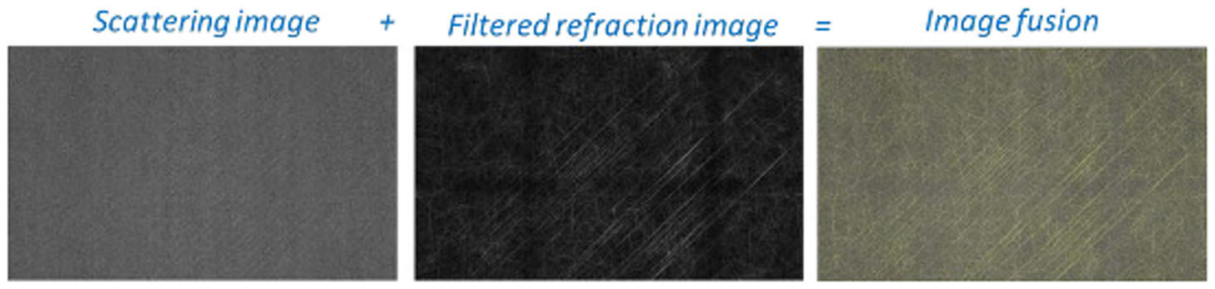

Fig. 11 Example of image fusion of the scattering image with the filtered scattering image for a composite sample with micro-cracks 


\section{Conclusion}

The EVITA demonstrator was presented and its performance was illustrated using two coupon samples with two types of artificial flaws: porosity and cracks. The flaws could be detected and quantified by the EVITA demonstrator with a comparably fast exposure time. The EVITA demonstrator was benchmarked against other state-of-the-art NDI methods. While phased array ultrasonic and through transmission ultrasonic were also able to detect the different porosity level, the ability of the EVITA demonstrator to quantify the level of porosity seems to be promising and will be further investigated in future research. Furthermore, while the detection of cracks can be readily achieved using the EVITA demonstrator and a geometrical filter, no other NDI method used for the benchmarking was able to detect the cracks induced in the structure. In combination with a relative fast exposure time (comparable to phased array ultrasonic), the EVITA demonstrator demonstrated unique features, which can benefit to the non-destructive inspection of lightweight materials such as composites.

The introduction of this innovative methodology is expected to provide the aeronautical industry with a reliable and detailed insight of the integrity of thin and thick composite structures as well as of complex geometry ones, such as integrated closed boxes and sandwiches. By increasing the level of detectability of defects in composite structures, as well as by detecting defects invisible to standard industrial nondestructive testing methodologies, the novel method will play a major role during the whole life cycle of composite components, reducing their inspection cost and increasing their reliability.

\section{Acknowledgments}

The authors acknowledge financial support from the European Union's Seventh Framework Programme for research technological development and demonstration under grant agreement n³14735.

Open Access This article is distributed under the terms of the Creative Commons Attribution 4.0 International License (http://creativecommons.org/licenses/by/4.0/), which permits unrestricted use, distribution, and reproduction in any medium, provided you give appropriate credit to the original author(s) and the source, provide a link to the Creative Commons license, and indicate if changes were made.

\section{References}

1. David, C., Nöhammer, B., Solak, H.H., Ziegler, E.: Differential x-ray phase contrast imaging using a shearing interferometer. Appl. Phys. Lett. 81, 3287-3289 (2002)

2. Atsushi, M., Shinya, K., Ichiro, K., Yoshitaka, H., Kengo, T., Yoshio, S.: Demonstration of X-Ray Talbot Interferometry. Jpn. J. Appl. Phys. 42, L866 (2003)

3. Pfeiffer, F., Weitkamp, T., Bunk, O., David, C.: Phase retrieval and differential phase-contrast imaging with low-brilliance X-ray sources. Nat. Phys. 2, 258-261 (2006)

4. Revol, V., Kottler, C., Kaufmann, R., Straumann, U., Urban, C.: Noise analysis of grating-based x-ray differential phase contrast imaging. Rev. Sci. Instrum. 81, 073709 (2010)

5. Revol, V., Plank, B., Kaufmann, R., Kastner, J., Kottler, C., Neels, A.: Laminate fibre structure characterisation of carbon fibre-reinforced polymers by X-ray scatter dark field imaging with a grating interferometer. NDT E Int. 58, 64-71 (2013) 
6. Revol, V., Kottler, C., Kaufmann, R., Neels, A., Dommann, A.: Orientation-selective X-ray dark field imaging of ordered systems. J. Appl. Phys. 112, 114903 (2012)

7. Jerjen, I., Revol, V., Brunner, A.J., Schuetz, P., Kottler, C., Kaufmann, R., et al.: Detection of stress whitening in plastics with the help of X-ray dark field imaging. Polym. Test. 32, 1094-1098 (2013)

8. Kottler, C., Revol, V., Kaufmann, R., Urban, C., Blanc, N., Niedermann, P., et al.: Recent developments on X-ray phase contrast imaging technology at CSEM. AIP Conf. Proc. 1466, 18-22 (2012)

9. Revol, V., Kisianos, K., Gresil, M., Tretout, H., Kanderakis, G., Sauer, M-O., et al.: "Non-destructive evaluation, inspection and testing of primary aeronautical composite structures using phase contrast X-ray imaging," presented at the 5th EASN International Workshop on Aerostructures, Manchester, UK (2015).

10. Gresil, M., Revol, V., Garcea, S. C., Chemama, R., Kanderakis, G., Kisianos, K., et al.: "Comparison between traditional non-destructive techniques and phase contrast X-Ray imaging applied to aeronautical carbon fibre reinforced polymer" In: 19th World Conference on Non-Destructive Testing Munich, Germany (2016).

11. Chantler, C.T.: Theoretical form factor, attenuation, and scattering tabulation for $Z=1-92$ from $E=1-10 \mathrm{eV}$ to $\mathrm{E}=0.4-1.0 \mathrm{MeV}$. J. Phys. Chem. Ref. Data 24, 71-643 (1995)

12. Henke, B.L., Gullikson, E.M., Davis, J.C.: X-Ray interactions: photoabsorption, scattering, transmission, and reflection at $\mathrm{E}=50-30,000 \mathrm{eV}, \mathrm{Z}=1-92$. At. Data Nucl. Data Tables 54, 181-342 (1993)

13. Weitkamp, T., Diaz, A., David, C., Pfeiffer, F., Stampanoni, M., Cloetens, P., et al.: X-ray phase imaging with a grating interferometer. Opt. Express 13, 6296-6304 (2005)

14. Yashiro, W., Terui, Y., Kawabata, K., Momose, A.: On the origin of visibility contrast in x-ray Talbot interferometry. Opt. Express 18, 16890-16901 (2010)

15. Revol, V., Jerjen, I., Kottler, C., Schütz, P., Kaufmann, R., Lüthi, T., et al.: Sub-pixel porosity revealed by xray scatter dark field imaging. J. Appl. Phys. 110, 044912 (2011) 